\title{
Sigmoidouterine Fistula: Anal Menstruation as a Rare Presentation
}

\author{
Shuchi Jain ${ }^{1}$, Shivi Jain ${ }^{2}$, Vaibhav Jain ${ }^{3}$ and Madhu Jain ${ }^{4}$ \\ 1,4 Department of Obstetrics \& Gynecology, Institute of Medical Sciences, \\ Banaras Hindu University, Varanasi,India \\ ${ }^{2}$ Department of Radiodiagnosis and Imaging, Institute of Medical Sciences, \\ Banaras Hindu University, Varanasi, India \\ ${ }^{3}$ Department of Plastic Surgery, Institute of Postgraduate Medical Education \\ \& Research, Kolkata,India
}

Correspondence should be addressed to: Madhu Jain; drmadhujainbhu@gmail.com

Received date: 5 February 2014; Accepted date: 6 March 2014; Published date: 18 July 2014

Academic Editor: Luis Guillermo Bahamondes

Copyright (C 2014. Shuchi Jain, Shivi Jain, Vaibhav Jain and Madhu Jain. Distributed under Creative Commons CC-BY 3.0

\begin{abstract}
Background: Anal menstruation due to sigmoidouterine fistula in the anterior wall of the uterus has not been reported so far. Case: A thirty year old female presented to us with periodical anal bleeding without natural menstrual loss one year after dilatation and curettage for 10 weeks' gestation. Hysterosalpingography raised the doubt of a fistulous tract between the uterus and the bowel with the dye present in the bowel.MRI revealed thinning of the anterior wall of the retroverted uterus with colon adherent to its anterior surface. Excision of the fistula along with a segment of sigmoid colon was done in a single stage. Conclusion: High degree of suspicion and planned investigations are required to reach at the diagnosis of such a bizarre condition.
\end{abstract}

Keywords: Fistulous communications, Sigmoidouterine fistula, Unnatural menstruation.

\section{Introduction}

Unsafe abortion is a global problem. The World Health Organization (2011) estimates that about 22 million abortions occur per year in the world under inadequate conditions and 14 unsafe abortions occur per thousand women. Hereby, we report the first case of 'anal menstruation' resulting from anteriorly placed sigmoidouterine fistula as a rare complication of unsafe abortion. A high index of suspicion and systematic workout may clinch the diagnosis. 


\section{Case}

A 30 year old female $(\mathrm{P} 3+1)$ presented with history of cyclical bleeding from anal canal for one year instead of regular bleeding per vagina ever since she had undergone dilatation \& evacuation by some untrained person for 10 weeks' gestation. Her Previous cycles were regular (per vaginal bleeding) with average flow since menarche. She gave no history of passage of faecal matter through vagina. She had no other complaint whatsoever during this period. The patient had soft abdomen with normal looking cervix, retroverted normal size uterus and clear, non-tender fornices. Digital rectal examination revealed a tender nodule present at the level of uterosacral ligament. Her hemogram and other biochemical parameters were within normal limits.

Trans-vaginal sonography (TVS) and Trans-rectal scanning showed a retroverted uterus with a short segment of sigmoid colon adherent to its anterior wall. Uterine myometrium was thinned out at this site with a suspicious track between the uterus and bowel loop suggesting a possibility of utero-sigmoid fistula. Sigmoidoscopy was inconclusive. Hysterosalpingography (HSG) was done with difficulty in passing the cannula, and it revealed contrast-opacified sigmoid colon and adjacent bowel loops. The uterus and the fallopian tubes could not be well appreciated (Fig.1).

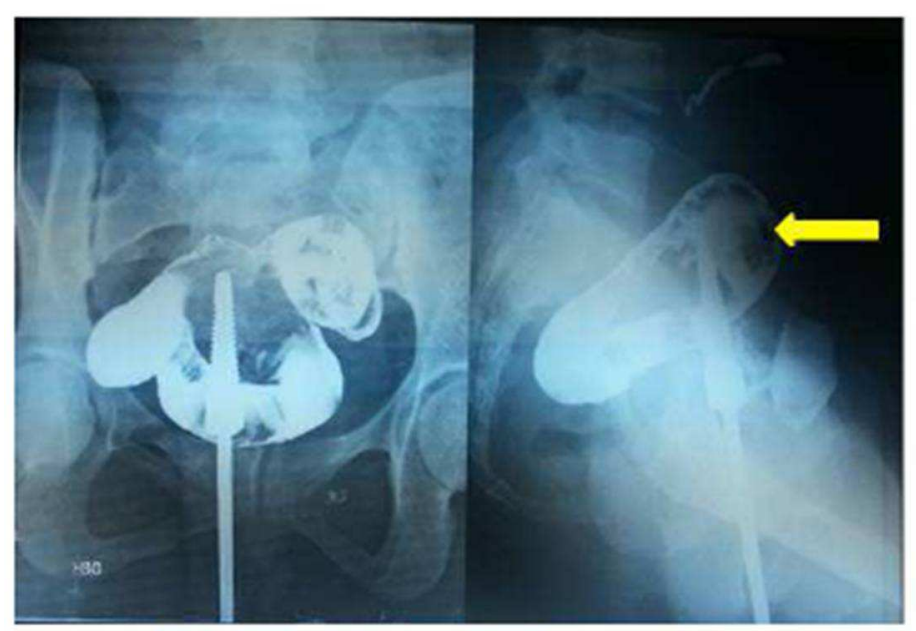

Figure 1: HSG (AP and lateral views) show contrast opacification of sigmoid colon (arrow) and adjacent bowel loop raising suspicion of presence of fistulous communication. Uterus and fallopian tubes could not be appreciated.

Barium enema (Fig.2 A \& B) demonstrated luminal narrowing with irregularity of the wall of proximal sigmoid colon at the site suspected of presence of fistulous communication on HSG. Sagittal-T2 weighted MR image showed thinning of myometrium in the anterior wall with the presence of ill-defined fat plane and adjacent sigmoid loop attached to the anterior surface of the uterus (Fig.3). It indicated the site of the fistulous tract. 

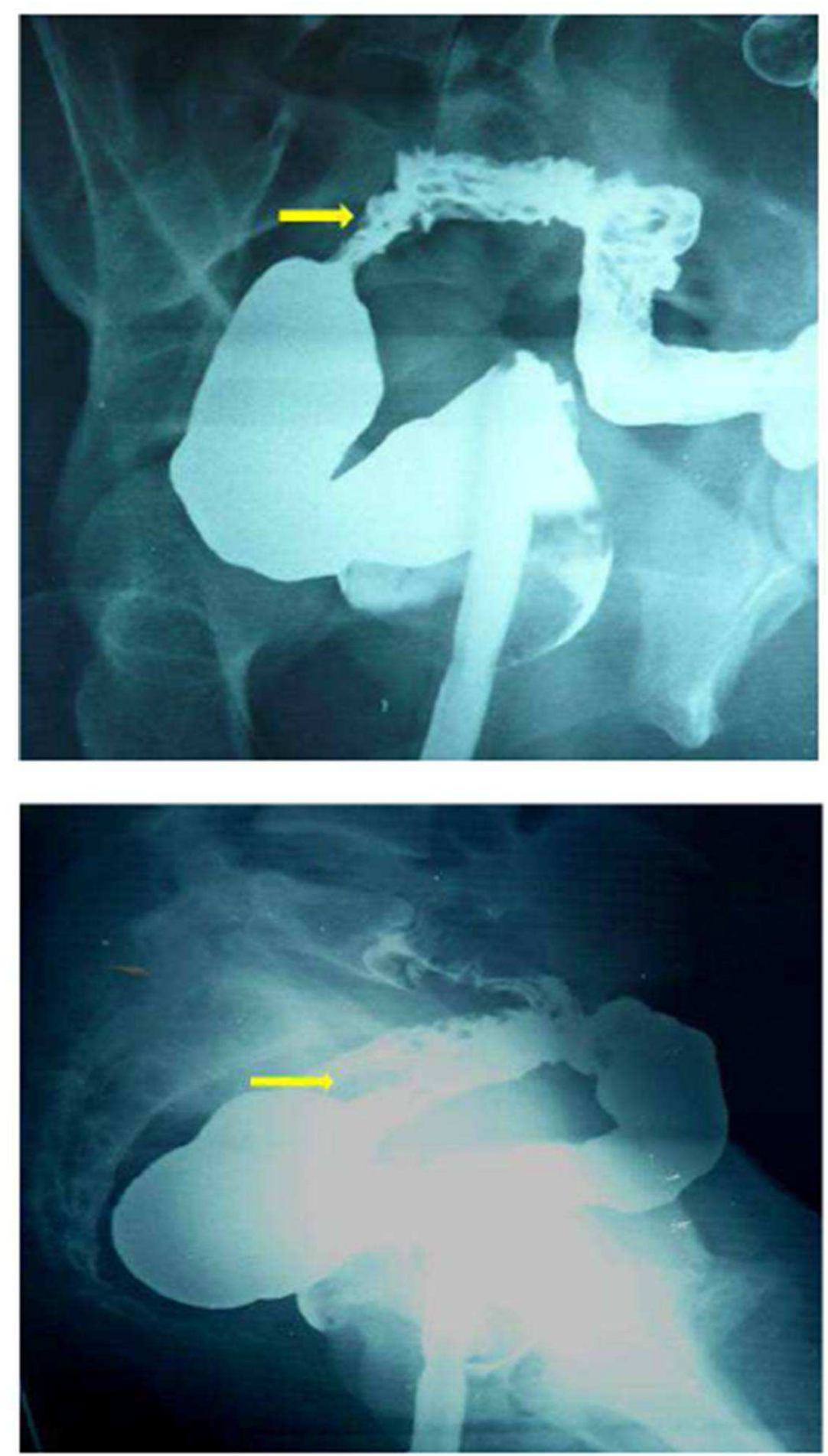

Figure 2: A (Oblique) \& 2B (Lateral View): Single contrast barium enema showing luminal narrowing with wall irregularity (arrows) of proximal sigmoid colon at site suspected of presence of fistulous communication during HSG. 


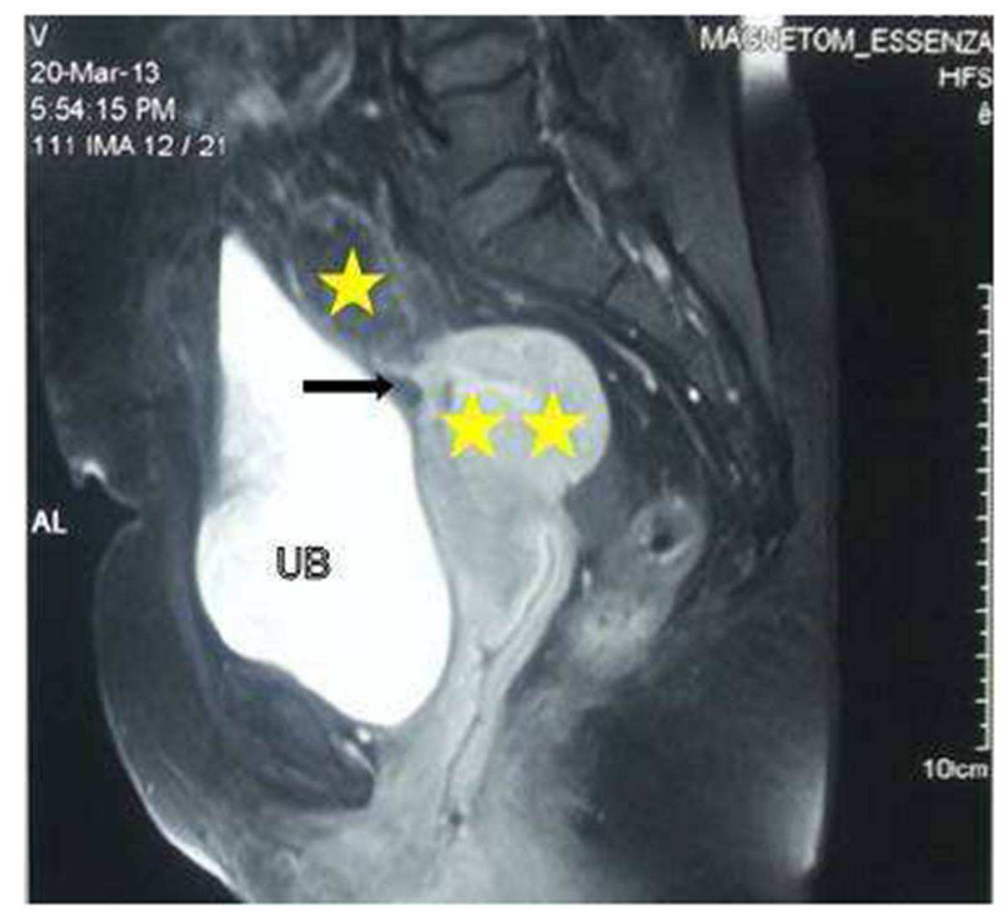

Figure 3: Sagittal T2 weighted MRI showing retroverted uterus (double asterisks) with presence of proximal sigmoid loop (single asterisk) in close approximation to anterior aspect of uterine body with loss of intervening fat plane (arrow). UB: Urinary bladder.

Exploratory laparotomy was performed and the sigmoid colon was found adherent to the anterior wall of the uterus (Fig.4).

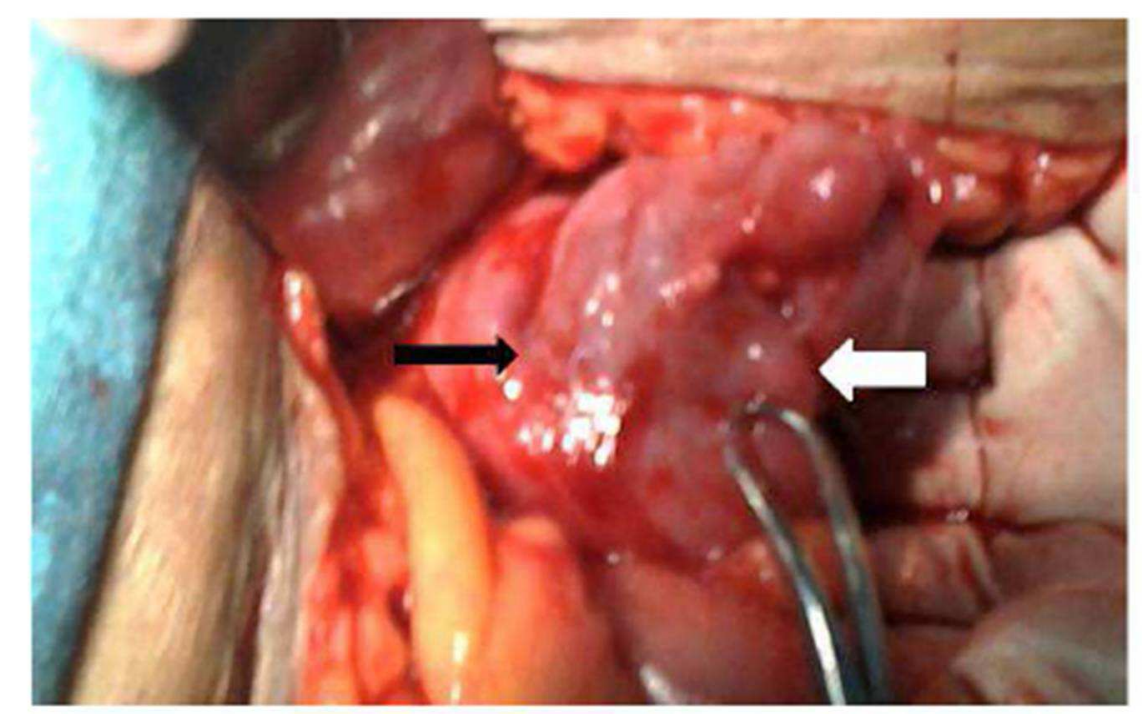

Figure 4: Sigmoid colon (black arrow) adherent to anterior surface of the uterus (white arrow). 
Their separation demonstrated the fistulous opening in the sigmoid colon (Fig.5).The tract was excised along with resection of a small segment of sigmoid colon because of inflammation and scarring, and the continuity of the colon was restored. The internal os was dilated with Hegar dilator under visual guidance to relieve the cervical stenosis. The uterine wound was closed, and bilateral tubal ligation was performed. The post-operative period was uneventful and the patient has normal menses since then. The resected specimen of bowel sent for histopathology revealed the presence of tubercular disease.

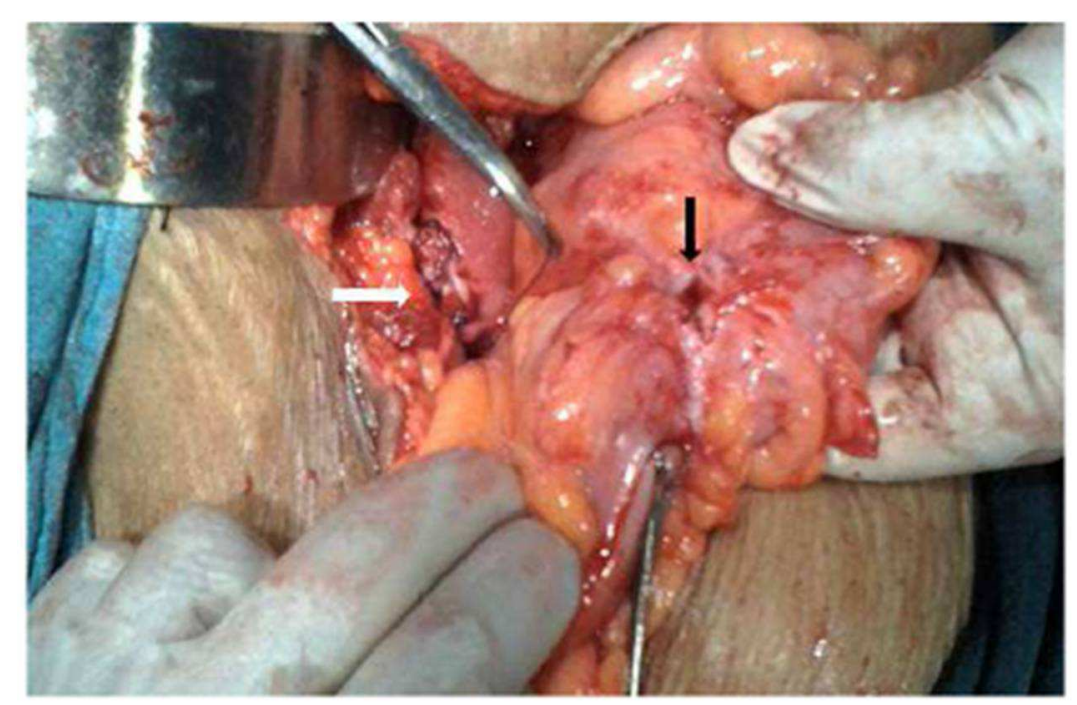

Figure 5: Opening of the fistula in the sigmoid colon (black arrow); the other end of the track in the uterus being closed (white arrow).

\section{Comment}

In spite of the legalization of abortions under the 'MTP Act' in India, it is not unusual to find them being performed by untrained or less trained persons, at times leading to serious maternal complications. 'Anal menstruation' as a result of sigmoidouterine fistula originating from the anterior surface of the uterus has hitherto been unheard of.

A colouterine fistula was first reported by Lejemtel in 1909 with three main etiologies under consideration: uterine trauma, an abscess rupturing into the bowel and the uterus, and uterine or sigmoid carcinoma. Mandato et al (2012) mention that so far, about twenty such cases have been reported in the literature.

Woods et al (1988) found that out of various possible unnatural connections between the genitourinary tract and the bowel, the colovesical fistula was the most common, followed by colovaginal fistula. The rarity of sigmoidouterine fistulas is probably explained by the fact that the uterus is a thick muscular organ, which poses obstacles for invasion for both benign and malignant disease. Colcock and Stahman (1972) suggested that such a fistula could result from the spontaneous rupture of gravid uterus, obstetric traumatic procedure such as curettage, uterine or colonic cancer, radiotherapy or an inflammatory process like diverticulitis and periappendicial abscess. Although the exact mechanism of development of sigmoidouterine fistula in our case is not very clear, it may be postulated that the inflammatory adherence of the bowel wall (rendered susceptible due to tuberculosis) to the uterus (which perforated during medical termination in unsafe hands) could 
have resulted in subsequent fistula formation.

Sigmoidouterine fistula may result into chronic vaginal discharge which may be purulent, hemorrhagic or fecal in nature. However, our patient presented with a very unusual symptom of 'anal menstruation' in the absence of periodical normal menses. Normally, one would have thought of hemorrhoids, large bowel pathology, and recto-sigmoid endometriosis etc. as a possible cause of such bleeding but the absence of normal menses altogether forced us to think of reverse flow of menstrual blood through the fistulous tract in the presence of cervical stenosis responsible for 'anal menstruation'. Cervical stenosis might have resulted from the trauma and infection associated with dilatation and curettage performed by an inexperienced person. The retroverted gravid uterus might have predisposed to injury to the anterior wall of the uterus while performing dilatation and curettage by an inexperienced hand. The reason of one way flow from the uterus to sigmoid colon and not the other way was perhaps due to thicker fecal contents of sigmoid colon as compared to menstrual blood and the distal obstruction in the uterus. This assumption gained strength from the fact that the patient started having natural menstruation after the cervical dilatation and excision of fistulous tract. Reverse flow of menstrual blood in adequate amount through the anus prevented the development of hematometra, hematosalpinx, and endometriosis. The absence of purulent or fecal discharge per vaginum could also result from cervical stenosis and tapering tract at the uterine end.

Many imaging modalities have been suggested and tried for establishing the diagnosis of sigmoidouterine fistula. Whereas CT scan can establish the diagnosis of diverticular disease, its role in demonstrating the fistula may be limited. Moreover, it is associated with a high dose of radiation. Beattie et al (2005) are of the opinion that multidetector computed tomography (MDCT) provides better visualisation of fistula, but it is associated with still greater radiation. Kassab et al (2008) have found MRI to be a more versatile, sophisticated and accurate diagnostic tool in showing the fistula. Huetter et al (1992) suggested that Charcoal Challenge Test could prove to be a non-invasive useful modality for the diagnosis of such an entity, but was not done by us as it cannot identify the exact site of the fistula and the tract. Sonohysterography is another modality for the diagnosis of such entity but needs costly contrast media.

Surgical treatment was a one-stage operation in our patient without sacrifice of the uterus or two stage colonic repair. En bloc, resection of the uterus and sigmoid colon is preferred if malignancy cannot be excluded. Hysterectomy may also be mandatory to extirpate a nidus of acute infection particularly in older woman who has completed her family. Severe local inflammation or obstruction of the bowel may necessitate a two-stage procedure involving resection and end colostomy, followed by re-anastomosis at a later date.

\section{Precis}

Anal menstruation as a result of sigmoidouterine fistula may complicate unsafe abortion and requires high degree of suspicion and planned investigations for proper management.

\section{Source of the work}

It was the patient admitted in the Obstetrics and Gynecology ward of Sir Sunderlal Hospital attached to the Institute of Medical Sciences, Banaras Hindu University, VARANASI.

No financial support was received for this report. There is no conflict of interest. 


\section{References}

1. Beattie, G.C., Nelson, M. and McMillen , I.M.(2005) "McMurray AH. Colouterine fistula mimicking pyometrium--diagnosis established with multi-detector computed tomography." Ulster Med J, 74(1), 51-53.

2. Colcock, B.P. and Stahman, F.D. (1972) "Fistula complicating diverticular disease of the sigmoid colon." Ann Surg, 175, 838846.

3. Huetter, P.C., Finkler, N.J. and Welch, W.R.(1992)" Colouterine fistula complicating diverticulitis: charcoal challenge test aids in diagnosis." Obstet Gynecol, 80(3 pt 2), 550-552.

4. Kassab, A., El-Bialy,G. and Hashesh, H.(2008) "Magnetic resonance imaging and hysteroscopy to diagnose colo-uterine fistula: a rare complication of diverticulitis." J Obstet Gynecol Res, 34, 117-120

5. Mandato,V.D.,Abrate,M. and Sandona, F.(2012) "Colouterine fistula complicating diverticulitis diagnosed at hysteroscopy: case report." J Minim Invasive Gynecol, 19(1),118-121

6. Vilallonga, R., Baena, J.A. and Fort, J.M. (2009) "Colouterine fistula complicating diverticulitis in elderly women." Int J Colorectal Dis, 24(5), 599-600.

7. WHO (2011), Unsafe Abortion: Global and Regional Estimates of the Incidence of Unsafe Abortion and Associated Mortality in 2008, sixth Ed.

8. Woods,R.J., Lavery,I.C. and Fazio, V.W.(1988) "Internal fistulas in diverticular disease". Dis. Colon Rectum, 31,591-596 